\title{
A Situational Analysis of Competences of Research Ethics Committee Members in Review of Research Protocols with Complex and New Study Designs in Uganda
}

\section{Provia Ainembabazi}

Infectious Diseases Institute https://orcid.org/0000-0003-2570-754X

\section{Barbara Castelnuovo}

Infectious Diseases Institute

\section{Stephen Okoboi}

Infectious Diseases Institute

Walter Joseph Arinaitwe

Infectious Diseases Institute

Rosalind Parkes-Ratanshi

Infectious Diseases Institute

Pauline Byakika-Kibwika ( $\square$ pbyakika@gmail.com )

Infectious Diseases Institute https://orcid.org/0000-0003-0757-1968

Research article

Keywords: Complex and New study designs, Research Ethics Committees, Competence

Posted Date: September 25th, 2020

DOI: https://doi.org/10.21203/rs.3.rs-66248/v1

License: (c) (i) This work is licensed under a Creative Commons Attribution 4.0 International License.

Read Full License 


\section{Abstract}

BACKGROUND Over the past two decades, Uganda has experienced a significant increase in clinical research driven by both industry and the need to combat the emergence and re-emergence of infectious disease epidemics. This has broadened the spectrum of research proposals that need evaluation by Research Ethics Committees (RECs) with associated requirement for new expertise. We assessed the competencies of REC members in review of research protocols with complex and new research study designs to guide development of a training curriculum to improve the quality of review.

METHODS This was a cross-sectional study design, with quantitative and qualitative data collection methods. We used a structured pre-coded questionnaire to collect data on competencies of REC members in review of research protocols with complex and new research study designs. Research Ethics Committee members were also asked to outline a list of additional topics for which they needed training. Data from coded questions was entered into Epidata Version 3.1 and then exported to STATA Version14.1 for analysis. Descriptive analysis was performed for quantitative data and findings were presented using percentages and frequencies.

RESULTS We enrolled 55/97 REC members from 6 RECs, majority of whom were males $(56.4 \%, n=$ 31/55). The level of competence for review of selected study design was lowest for Controlled Human Infection Model $(6,10.9 \%)$ and reverse pharmacology design $(6,10.9 \%$,), and highest for cluster randomized study design $(29,52.7 \%)$ and implementation science research $(29,52.7 \%)$.

CONCLUSION There is lack of competence in review of research protocols with complex and new study design and our analysis suggests that additional training in this area is an urgent priority.

\section{Background}

Clinical research remains cardinal in advancing our knowledge of disease, human biology and behavior and informing our health care practice(1). There has been a surge in clinical research driven by both industry and the need to combat the emergence and re-emergence of infectious disease epidemics in Uganda, including HIV, hemorrhagic fevers, tuberculosis, malaria, neglected tropical diseases, noncommunicable diseases and the recent Severe Acute Respiratory Syndrome Corona Virus-2 that causes Corona virus disease-19 (COVID-19)(2-5). This has broadened the spectrum of research activities in the quest for solutions to improve health and wellbeing. Clinical research, especially that involve invasive procedures and interventions, may carry risks to human health and safety or may compromise the rights, values and interests of research participants or volunteers(6). Nonetheless, human participation in clinical research is important, and every effort should be made to minimize harm to research participants(6).

With the introduction of antiretroviral therapy, scientists in Uganda embarked on clinical trials with adherence and long-term efficacy monitoring $(7,8)$. In the recent past; there has been increasing interest in HIV vaccines research and investigations into a cure for HIV (9). These research interests to develop 
novel preventive, investigational and treatment strategies need to be supported by efficient RECs to review the science and ethics, approve and monitor research regulatory compliance with assurance for safety and wellbeing of humans as research participants.

Members of RECs review the science and ethics of research proposals, approve research protocols and oversee the conduct of clinical research with the aim of minimizing risk to humans and ensuring respect for the research participant's rights, values and interests (10).

It is therefore important, to ensure that members of RECs have the competence to review research protocols to protect the safety, rights and welfare of research participants while advancing knowledge through high quality research. Therefore, this study aimed to assess the competencies of REC members in review of research protocols with complex and new research study designs, in order to guide development of relevant training curriculum for REC members.

\section{Methods}

\section{Study design, site and population}

This was a cross-sectional study among REC members from 6 accredited RECs located in MakerereMulago Hospital complex. The RECs include; School of Health Sciences REC (SHSREC), School of Medicine REC(SOMREC), School of Biomedical Sciences REC (SBSREC), School of Public Health Sciences REC (SPHREC), Mulago Hospital REC (MHREC) and Uganda Cancer Institute REC (UCIREC). School of Health Sciences REC, SOMREC, SBSREC, SPHREC, MHREC and UCIREC comprise of 14, 20, 16, 21,13 and 17 REC members respectively. All REC members were eligible to enrol into the study if they were willing to participate.

\section{Study procedure}

The REC Chairs were contacted and informed about the study. The list of REC members was obtained from the REC administrators with contact details: email and phone number. Convenience sampling was used to enrol participants. Participants were selected based on availability and willingness to take part in the assessment. A notice was sent to each participating REC member and an appointment for the study interview made. Written informed consent form was obtained from the participants before the conduct of the face-to-face interview. However, due to the COVID-19 lock-down restriction measures enforced by the Uganda Ministry of Health, the data collection method was changed from face-face paper basedquestionnaire to an online survey using KoBoToolbox. An email with a link to the online Informed Consent Form and the survey were sent to REC members inviting them to participate in the survey. In order to increase on the response rate, email and telephonic reminders were sent for participants who had not filled the survey after 7 and at 14 days of sending the survey.

\section{Data collection}


Data were collected using a pre-coded questionnaire developed for this study in English language. The questionnaire consisted of demographic characteristics and questions on competence in review of research protocols with complex and new study designs (adaptive, ecological, phase I, phase II, implementation science, step wedged design, cluster randomized design, controlled human infection model, reverse pharmacological design, and evaluation of studies with new technology/devices). The questions assessing the competence were on a Likert scale with four alternative responses with $1=$ not competent, $2=$ somewhat competent, $3=$ competent, $4=v e r y$ competent. We used open-ended questions to collect data on other study designs that were not included on the list and a list of additional topics for which they needed training. The study coordinator checked the complete survey questionnaires for completeness.

\section{Data management and analysis}

Data was entered into the study data base developed using Epi data version 3.1 released by Epi data Association, Odense, Denmark with in-built quality control checks. The final dataset was exported to STATA version 14.1 released by StataCorp for analysis. The competence of REC members were further categorized into two categories: competent (competent and very competent) and not competent (not competent and somewhat competent). Descriptive analysis was done and findings were summarized using frequencies and percentages.

\section{Ethical consideration}

The REC chairpersons were informed about the survey before administering the survey to REC members. Informed consent was obtained before administering the questionnaire. The study was approved by School of Medicine Research Ethics Committee (\#REC REF 2020-024) and Uganda National Council for Science and Technology (HS542ES).

\section{Results}

\section{Social demographic characteristics of the participants}

A total of 55 out of the 97 contacted REC members filled the survey giving a $56.7 \%$ response rate. All REC members $(23 / 23,100 \%)$ that were contacted physically completed the survey. A total of 32 out of 74 REC members contacted through email completed the survey. Of the 55 respondents, $56.4 \%$ were male. Participants had diverse expertise in the areas of social sciences, bioethics, epidemiology and biostatistics, psychology, dentistry, education, medicine, oncology, public health, basic sciences, nursing and pharmacy as shown in table 1 below. Majority of the participants had attained a PhD degree (31/55, $56.4 \%$ ). School of Health sciences REC had the highest number of participants (14, 25.5\%).

\section{Competency in Review of Research Protocols with Complex and New Study Designs}

Generally, most of the REC members reported they were not competent in reviewing research protocols with complex and new study designs. The level of competence for review of selected complex and new 
study designs was lowest for controlled human infection model $(6,10.9 \%)$, reverse pharmacology design $(6,10.9 \%)$ and highest for cluster randomized study design $(29,52.7 \%)$, implementation science research $(29,52.7 \%)$ as shown in figure 1 .

\section{Additional Areas that Pause Challenges during Review of Research Protocols}

Some REC members reported difficulty in review of research protocols for genetic studies; studies involving longitudinal large data, Investigation New Drug applications and New technology/devices. They also reported difficulty in review of herbal medicine research as well as management of ethical dilemmas that arise with complex and new study designs. 
Table 1

Demographic characteristics of the participants

\begin{tabular}{|c|c|}
\hline Variable & $\begin{array}{l}\text { Frequency (percentage) } \\
(\mathrm{N}=55)\end{array}$ \\
\hline \multicolumn{2}{|l|}{ Sex } \\
\hline Male & $31(56.4)$ \\
\hline \multicolumn{2}{|l|}{ Highest level of education } \\
\hline Certificate & $01(01.8)$ \\
\hline Diploma & $01(01.8)$ \\
\hline Bachelors & $01(01.9)$ \\
\hline Masters & $21(38.2)$ \\
\hline PHD & $31(56.4)$ \\
\hline \multicolumn{2}{|l|}{ REC membership } \\
\hline SHSREC & $14(25.5)$ \\
\hline SOMREC & $09(16.4)$ \\
\hline SBSREC & $08(14.6)$ \\
\hline SPHREC & $09(16.4)$ \\
\hline UCIREC & $08(14.6)$ \\
\hline MHREC & $07(12.7)$ \\
\hline \multicolumn{2}{|l|}{ Area of expertise } \\
\hline Medicine & $11(20.0)$ \\
\hline Nursing & $03(5.5)$ \\
\hline Social sciences & 05(09.1) \\
\hline Bioethics & $04(07.3)$ \\
\hline Basic sciences & $06(10.9)$ \\
\hline Public health & $15(27.3)$ \\
\hline Epidemiology and Biostatistics & $04(07.3)$ \\
\hline Dentistry & 02(03.6) \\
\hline Psychology & $01(01.8)$ \\
\hline Education & $02(03.6)$ \\
\hline
\end{tabular}




\section{Discussion}

\begin{tabular}{|ll|}
\hline Variable & $\begin{array}{l}\text { Frequency (percentage) } \\
(\mathbf{N}=\mathbf{5 5})\end{array}$ \\
\hline Pharmacy & $02(03.6)$ \\
\hline
\end{tabular}

We assessed the competencies of REC members in review of research protocols with complex and new research study designs to guide development of a training curriculum to improve the quality of review. The response rate amongst REC members in this study was slightly higher compared to $52 \%$ in a similar study in a similar setting (11). The ratio of male to female participants was almost equal, which could be attributed to the guidelines by the Uganda National council for Science and Technology which encourage RECs to have diverse membership, including consideration of gender(10). Majority of the participants had a master's degree or higher qualification. This is attributed to the national guidelines requirement that the REC to be sufficiently qualified through the experience and expertise in different specialty areas (10).

Most participants reported that they were not competent to review research protocols with complex and new study designs. Our findings are in agreement with the discussion highlighted during the 10th Annual National Research Ethics Conference, that emergence of new study designs like Controlled Human Infection Model, studies with digital intervention and genetic studies present enormous scientific and ethical challenges for review by REC members(12-15). The lack of competence in review of research protocols with complex and new study design could lead to a longer or delayed research review process, poor quality review and rejection of important studies. The lack of competence reported could be due to broadened spectrum of research emanating from significant increase in clinical research that is driven by the changing disease patterns.

\section{Limitations Of The Study}

There may be some possible limitations of this study. The small sample size which we attribute to the impact of COVID-19 restrictive measures affected our analysis and conclusions. In addition, the RECs involved are situated in the capital city of Uganda, Kampala, where most research institutions are located thus the results may not be generalisable to all RECs in the country due to differences in the volumes and scope of the research received by these RECs and the academic environment.

\section{Conclusion And Recommendation}

There is lack of competence in review of complex and new study design among the REC members studied and additional training in this area is an urgent priority. Results of this study will inform development of a training curriculum for REC members in Uganda.

\section{List Of Abbreviations}


REC Research Ethics Committee

SOMREC School of Medicine Research Ethics Committee

SHSREC School of Health Sciences Research Ethics Committee

MHREC Mulago Hospital Research Ethics Committee

UCIREC Uganda Cancer Institute Research Ethics Committee

SBSREC School of Biomedical Sciences Research Ethics Committee

SPHREC School of Public Health Research Ethics Committee

COVID-19 Corona Virus Disease-19

\section{Declarations}

Ethics approval and consent to participate: The study received ethical approval from School of Medicine Research Ethics Committee (\#REC REF 2020-024) and Uganda National Council for Science and Technology (HS542ES).

Written consent was obtained before the survey was undertaken for the surveys that were collected using a paper-based form of data collection. For the online survey, the e-consent form provided detailed information about the survey. Individuals who voluntarily choose to participate clicked on a box "I agree to participate" and those not willing will click on "I do not agree to participate". E-consent was not taken as a "full signature" but rather an indication of accepting to participate in the survey.

Consent for publication: Not applicable. The manuscript does not contain data from any individual person's data.

Availability of data and materials: The datasets used and/or analyzed during the current study are available from the corresponding author on a reasonable request. All data generated or analyzed during this study are included in this published article.

Competing interests: Authors declare that there are no competing interests

Funding: The study is supported by the Ethics project entitled "Towards eliminating HIV in Uganda by 2030; Preparing Ethical Review Committees to support this agenda" at the Infectious Diseases Institute, which is, funded the National Institutes of Health (1 G11 TW011309-01). The funding bodies did not have a role in the design of the study and collection, analysis, and interpretation of data and in writing the manuscript.

\section{Authors' contributions:}


AP Participated in writing the protocol, acquiring regulatory approvals and data collection, performed the analysis, drafted, revised and finalised the manuscript

$\mathrm{BC}$ conceptualised the idea, participated in writing the protocol, revised and finalised the manuscript So Conceptualised the idea, revised and finalised the manuscript

AJW Conceptualised the idea, revised and finalised the manuscript

RPR Conceptualised the idea, participated in writing the protocol, revised and finalised the manuscript

PBK Conceptualised the idea, participated in writing the protocol, acquiring regulatory approvals and data collection, cross checked the analysis, revised and finalised the manuscript

All authors read and approved the final manuscript

Acknowledgements: We would like to thank the REC members who participated in the study. We would like to extend our sincere gratitude to the research office; Infectious Diseases Institute for the support rendered particularly Paul K. Gonza, the research Manager, Nabukenya Sylvia and Kisekka Faith, the Regulatory officers.

\section{Authors' information (optional):}

${ }^{1}$ Infectious Diseases Institute, Makerere University, College of Health Sciences P.O BOX 22418, Kampala, Uganda.

${ }^{2}$ Department of Medicine, Makerere University College of Health Sciences,

Kampala, Uganda PO BOX 7072, Kampala

\section{References}

1. Novitzke JM. The significance of clinical trials. J Vasc Interv Neurol. 2008;1(1):31-.

2. Andrews SM, Rowland-Jones S. Recent advances in understanding HIV evolution. F1000Res. 2017;6:597-.

3. Carter R, Mendis KN. Evolutionary and historical aspects of the burden of malaria. Clin Microbiol Rev. 2002;15(4):564-94.

4. Fhogartaigh CN, Aarons E. Viral haemorrhagic fever. Clin Med (Lond). 2015;15(1):61-6.

5. Benvenuto D, Giovanetti M, Ciccozzi A, Spoto S, Angeletti S, Ciccozzi M. The 2019-new coronavirus epidemic: Evidence for virus evolution. Journal of medical virology. 2020;92(4):455-9.

6. Good clinical practice research guidelines reviewed, emphasis given to responsibilities of investigators: second article in a series. J Oncol Pract. 2008;4(5):233-5. 
7. Dolgin E. Long-acting HIV drugs advanced to overcome adherence challenge. Nature Medicine. 2014;20(4):323-4.

8. Spreen WR, Margolis DA, Pottage JC, Jr. Long-acting injectable antiretrovirals for HIV treatment and prevention. Current opinion in HIV and AIDS. 2013;8(6):565-71.

9. Pavlakis GN, Felber BK. A new step towards an HIV/AIDS vaccine. Lancet. 2018;392(10143):192-4.

10. UNCST. National guidelines for research involving humans as research participants: Uganda National Council for Scince and Technology Kampala-Uganda; 2014.

11. Ibingira BR, Ochieng J. Knowledge about the research and ethics committee at Makerere University, Kampala. Afr Health Sci. 2013;13(4):1041-6.

12. Nelson NF. Emerging ethical issues: experiences of the FRECU. 10th Annual National Research Ethics Conference; Serena Conference center, Kampala: Uganda National Council for Science and Technology; 2018.

13. David Kaawa-Mafigiri DE-S, editor Genetic research in African populations:societal perspective, emerging ethical issues, challenges and opportunities. 10th Annual National Research Ethics Conference; 2018; Serena conference hall, Kampala: Uganda National Council for Science and Technology.

14. Mfutso-Bengo J, editor Ethics, Acceptability \& Feasibility of Controlled Human Infection Models (CHIM) in Uganda/africa. 10th Annual Research Ethics Conference; 2018; Serena conference hall, Kampala: Uganda National Council for Science and Technology.

15. Kakure WBNENW, editor Proceedings of the 10th Annual Research Ethics Conference. 10th Annual Research Ethics Conference; 2018; Serena conference hall, kampala: Uganda National Council for Science and Technology.

\section{Figures}

\section{Image not available with this version}

\section{Figure 1}

Proportion of REC members competent in review of complex and new study designs. 


\section{Supplementary Files}

This is a list of supplementary files associated with this preprint. Click to download.

- supplement1.docx

- supplement2.pdf 